Reference System, Germany

Solar Combisystem for Single-Family House

INFO Sheet A09

Description:

Date:

Authors:

Download possible at:
Definition of reference solar combisystem, Germany

23.03.2018, revised $10.04 .2018^{1}$

Stephan Bachmann (ITW Stuttgart), Stephan Fischer (ITW Stuttgart), Bernd Hafner (RHC-Platform)

http://task54.iea-shc.org/

\title{
Introduction
}

This document describes the reference solar combisystem for domestic hot water preparation and space heating in Germany. The system is modelled with TRNSYS to calculate the fuel consumption and electric energy needed to provide the required domestic hot water and space heating as well as the substituted fuel provided by the combisystem. Using this result the levelized costs of heating (LCoH) for the substituted fuel is calculated using Eq. 1 and the reference costs for the investment of the system, installation costs, fuel and electricity costs.

\section{Hydraulic Scheme of the System}

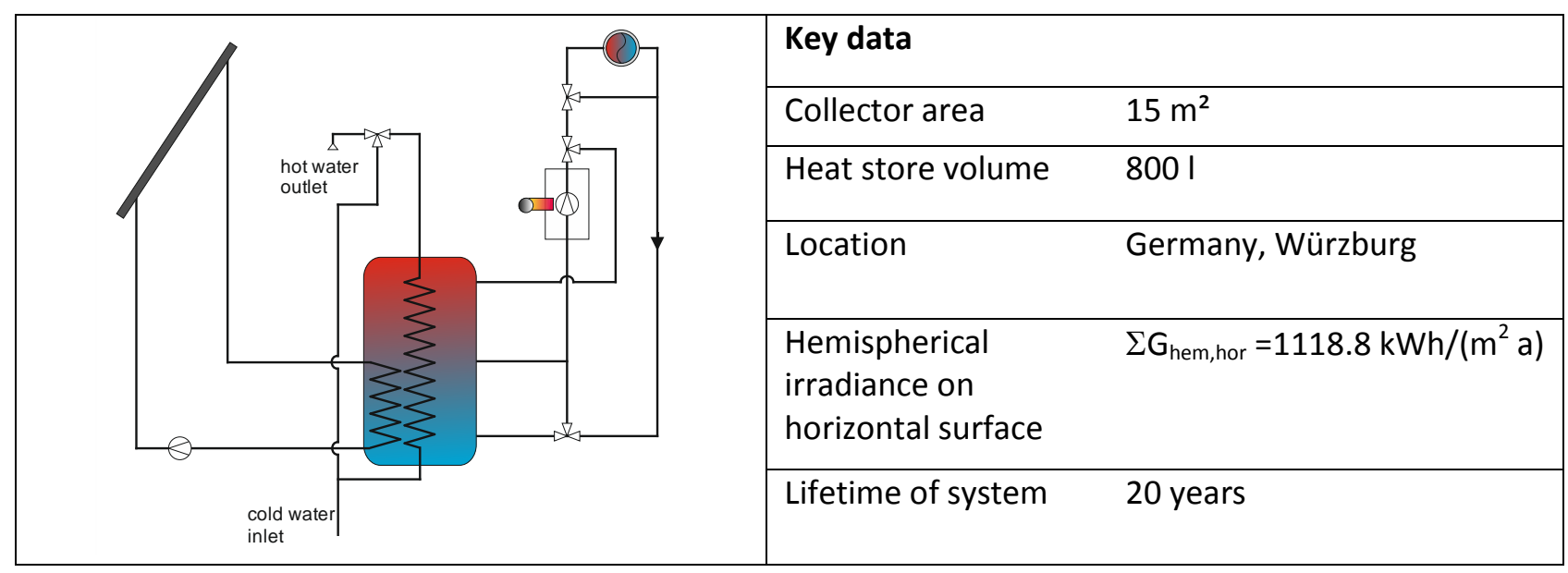

\section{Levelized Cost of Heat (LCoH)}

\begin{tabular}{|l|l|}
\hline LCoHs solar part without VAT & $0.206 € / \mathrm{kWh}$ \\
\hline LCoHc conventional part without VAT & $0.119 € / \mathrm{kWh}$ \\
\hline LCoHo complete system without VAT & $0.140 € / \mathrm{kWh}$ \\
\hline
\end{tabular}


Reference System, Germany

Solar Combisystem for Single-Family House

INFO Sheet A09

\section{Details of the System}

\begin{tabular}{|c|c|}
\hline Location & Germany, Würzburg \\
\hline Type of system & combisystem \\
\hline $\begin{array}{l}\text { Weather data including } \\
\text { - Hemispherical irradiance on horizontal surface } \\
\text { - Beam irradiance on horizontal surface } \\
\text { - Diffuse irradiance on horizontal surface } \\
\text { - Ambient temperature } \\
\text { in hourly values }\end{array}$ & $\begin{array}{l}\text { test reference year (TRY Würzburg) } \\
\Sigma G_{\text {hem,hor }}=1118.8 \mathrm{kWh} /\left(\mathrm{m}^{2} \mathrm{a}\right) \\
\Sigma \mathrm{G}_{\text {beam,hor }}=550.1 \mathrm{kWh} /\left(\mathrm{m}^{2} \mathrm{a}\right) \\
\left.\Sigma \mathrm{G}_{\text {diff,hor }}=568.7 \mathrm{kWh} / \mathrm{m}^{2} \mathrm{a}\right) \\
\mathrm{T}_{\text {amb,av }}=9.0^{\circ} \mathrm{C}\end{array}$ \\
\hline $\begin{array}{l}\text { Collector orientation } \\
\text { - Collector tilt angle to horizontal } \\
\text { - South deviation of collector } \\
\text { - Ground reflectance } \\
\text { - Resulting hemispherical irradiance on tilted } \\
\text { surface }\end{array}$ & $\begin{array}{l}45^{\circ} \\
\text { south }=0^{\circ} \\
0.2 \\
\Sigma G_{\text {hem,titt }}=1229.8 \mathrm{kWh} /\left(\mathrm{m}^{2} \text { a) }\right.\end{array}$ \\
\hline $\begin{array}{l}\text { Load information including } \\
\text { - Heat demand space heating } \\
\text { - Tapping profile } \\
\text { - Store heat losses } \\
\text { - Tapping temperature } \\
\text { - Average inlet temperature of cold water } \\
\text { - Cold water inlet temperature amplitude }\end{array}$ & $\begin{array}{l}9090 \mathrm{kWh} / \mathrm{a} / 1 / \\
\text { EU-tapping profile L ( } 4254 \mathrm{kWh} / \mathrm{a}) / 2 / \\
2041 \mathrm{kWh} \\
55^{\circ} \mathrm{C} \text { according EU tapping profile } \\
10^{\circ} \mathrm{C} \\
0 \mathrm{~K}\end{array}$ \\
\hline Collector information based on gross area & TRNSYS-type 132 \\
\hline Number of collectors & 6 \\
\hline Collector area of one collector & $2.5 \mathrm{~m}^{2}$ \\
\hline Maximum collector efficiency & 0.684 \\
\hline Incidence angle modifier for direct irradiance $b_{0}$ & 0.2 \\
\hline Incidence angle modifier for diffuse Irradiance & 0.91 \\
\hline Linear heat loss coefficient & $3.51 \mathrm{~W} /\left(\mathrm{m}^{2} \mathrm{~K}\right)$ \\
\hline 2nd order heat loss coefficient & $0.011 \mathrm{~W} /\left(\mathrm{m}^{2} \mathrm{~K}^{2}\right)$ \\
\hline Effective heat capacity & $8.0 \mathrm{~kJ} /\left(\mathrm{m}^{2} \mathrm{~K}\right)$ \\
\hline Heat store parameters & TRNSYS-type 340 \\
\hline Heat store volume & 8001 \\
\hline Auxiliary volume for DHW preparation & 4241 \\
\hline Store inner diameter & 0.79 \\
\hline Rel. height of solar inlet & 0.4 \\
\hline Rel. height of solar outlet & 0.04 \\
\hline Rel. height of auxiliary inlet & 0.9 \\
\hline Rel. height of auxiliary outlet & 0.47 \\
\hline Rel. height of space heating inlet & 0.2 \\
\hline Rel. height of space heating outlet & 0.45 \\
\hline Rel. height of cold water inlet & 0.05 \\
\hline Rel. height of hot water outlet & 0.95 \\
\hline
\end{tabular}


Reference System, Germany

Solar Combisystem for Single-Family House

INFO Sheet A09

\begin{tabular}{|c|c|}
\hline Rel. height of sensor for aux. charging & 0.6 \\
\hline $\begin{array}{l}\text { Rel. height of sensor for space heating } \\
\text { preheating }\end{array}$ & 0.45 \\
\hline Rel. height of sensor for collector loop & 0.2 \\
\hline Set temperature for DHW & $62.5^{\circ} \mathrm{C}$ \\
\hline $\begin{array}{l}\text { Temperature difference space heating preheat } \\
\text { on }\end{array}$ & $4 \mathrm{~K}$ \\
\hline $\begin{array}{l}\text { Temperature difference space heating preheat } \\
\text { off }\end{array}$ & $2 \mathrm{~K}$ \\
\hline Overall heat loss capacity rate of store & $4.4 \mathrm{~W} / \mathrm{K}$ \\
\hline Effective vertical conductivity & $1.2 \mathrm{~W} /(\mathrm{mK})$ \\
\hline Heat transfer capacity rate of solar loop HX & $(\mathrm{kA})_{\mathrm{WT}, \mathrm{Sol}}=165,9 \cdot \dot{\mathrm{m}}^{0,283} \cdot \vartheta_{\mathrm{m}}{ }^{0,524}[\mathrm{~W} / \mathrm{K}]$ \\
\hline Heat transfer capacity rate of hot water HX & $(\mathrm{kA})_{\mathrm{WT}, \mathrm{HW}}=75,8 \cdot \dot{\mathrm{m}}^{0,252} \cdot \vartheta_{\mathrm{m}}^{1,026}[\mathrm{~W} / \mathrm{K}]$ \\
\hline Volume solar loop HX & 11.81 \\
\hline Volume hot water $\mathrm{HX}$ & 38.51 \\
\hline Maximum heat store temperature & $90^{\circ} \mathrm{C}$ \\
\hline Ambient temperature of heat store & $15^{\circ} \mathrm{C}$ \\
\hline \multicolumn{2}{|l|}{ Solar thermal controller and hydraulic piping } \\
\hline Total pipe length of collector loop & $20 \mathrm{~m}$ \\
\hline Inner diameter of collector loop pipe & $20 \mathrm{~mm}$ \\
\hline Temperature difference collector start-up & $6 \mathrm{~K}$ \\
\hline Temperature difference collector shut-off & $4 \mathrm{~K}$ \\
\hline Electric power of solar thermal controller & $3 \mathrm{~W}$ \\
\hline $\begin{array}{l}\text { Operating hours of solar thermal controller per } \\
\text { year }\end{array}$ & $8760 \mathrm{~h}$ \\
\hline Electric consumption of controller per year & $26.3 \mathrm{kWh}$ \\
\hline Electric power of solar loop pump & $55 \mathrm{~W}$ \\
\hline Operating hours of solar loop pump & $1073 \mathrm{~h}$ \\
\hline Electric consumption of solar loop pump & $59 \mathrm{kWh}$ \\
\hline Electric consumption of other el. components & - \\
\hline \multicolumn{2}{|l|}{ Conventional system } \\
\hline Type of auxiliary heating & Gas condensing boiler \\
\hline Boiler capacity & $19 \mathrm{~kW}$ \\
\hline Mass flow & $1090 \mathrm{~kg} / \mathrm{h}$ (delT = $15 \mathrm{~K})$ \\
\hline Efficiency factor of boiler & 0.9 \\
\hline Electric power of controller & $3 \mathrm{~W}$ \\
\hline Operating hours of controller per year & 8760 \\
\hline Electric consumption of controller per year & $26.3 \mathrm{kWh}$ \\
\hline Electric power of pump & $55 \mathrm{~W}$ \\
\hline $\begin{array}{l}\text { Operating hours of pump (aux. heating + space } \\
\text { heating) }\end{array}$ & $3987 \mathrm{~h}$ \\
\hline Electric consumption of pump per year & $219 \mathrm{kWh}$ \\
\hline Investment costs $I_{0}$ & $5500 €$ \\
\hline
\end{tabular}


Reference System, Germany

Solar Combisystem for Single-Family House

INFO Sheet A09

\begin{tabular}{|c|c|}
\hline Investment costs solar thermal system & \\
\hline $\begin{array}{l}\text { Solar thermal collector, heat store, solar } \\
\text { thermal controller solar thermal hydraulic } \\
\text { components }\end{array}$ & $8000 € / 5 /$ \\
\hline Installation & $2000 € / 5 /$ \\
\hline $\begin{array}{l}\text { Credit conventional heat store and share of } \\
\text { installation }\end{array}$ & $-1000 €$ \\
\hline Overall investment costs solar thermal part $I_{0}$ & $9000 €$ \\
\hline \multicolumn{2}{|l|}{ Operation costs conventional part per year } \\
\hline Auxiliary heat demand hot water & $3743 \mathrm{kWh} / \mathrm{a}$ \\
\hline Fuel demand hot water & $4159 \mathrm{kWh} / \mathrm{a}$ \\
\hline Heat demand space heating & $7506 \mathrm{kWh} / \mathrm{a}$ \\
\hline Fuel demand space heating & $8340 \mathrm{kWh} / \mathrm{a}$ \\
\hline Fuel demand hot water + space heating $E_{t}$ & $12499 \mathrm{kWh} / \mathrm{a}$ \\
\hline Cost per kWh fuel (gas) & $0.066 € \mathrm{kWh} / \mathrm{a} / 4 /$ \\
\hline Fuel costs & $825 € / a$ \\
\hline Electricity demand & $246 \mathrm{kWh} / \mathrm{a}$ \\
\hline Cost per kWh electric energy /4/ & $0.254 €$ \\
\hline Electricity costs & $62 € / a$ \\
\hline Maintenance costs & $200 € / a / 3 /$ \\
\hline Gas meter & $130 € / a / 3 /$ \\
\hline $\begin{array}{l}\text { Yearly operation and maintenance cost } \\
\text { conventional part } C_{t}\end{array}$ & $1217 €$ \\
\hline \multicolumn{2}{|l|}{ Operation costs solar part per year } \\
\hline Electricity demand & $85 \mathrm{kWh} / \mathrm{a}$ \\
\hline Cost per kWh electric energy /4/ & $0.254 €$ \\
\hline Electricity costs & $22 € / a$ \\
\hline Maintenance costs $\left(I_{0} * 2 \%\right)$ & $180 € / a$ \\
\hline $\begin{array}{l}\text { Yearly operation and maintenance cost solar } \\
\text { part } C_{t}\end{array}$ & $202 € / a$ \\
\hline $\begin{array}{l}\text { Fractional energy savings with credit for } 150 \text { - } \\
\text { store, } U A=2,05 \mathrm{~W} / \mathrm{K}\end{array}$ & $20.2 \%$ \\
\hline Saved final energy (year t) $E_{t}$ & $3162 \mathrm{kWh}$ \\
\hline Type of incentives & None \\
\hline Amount of incentives & $0 €$ \\
\hline Lifetime of system & 20 year \\
\hline Discount rate $r$ & $0 \%$ \\
\hline Inflation rate & $0 \%$ \\
\hline Corporate tax rate TR & $0 \%$ \\
\hline Asset depreciation (year t) $\mathrm{DEP}_{\mathrm{t}}$ & $0 €$ \\
\hline $\begin{array}{l}\text { Subsidies and incentives (year } \mathrm{t} \text { ) } \mathrm{S}_{\mathrm{t}} \text { (considered } \\
\text { in } \mathrm{I}_{0} \text { ) }\end{array}$ & $0 €$ \\
\hline
\end{tabular}


Reference System, Germany

Solar Combisystem for Single-Family House

INFO Sheet A09

\begin{tabular}{|l|l|}
\hline Residual value RV & $0 €$ \\
\hline Discount rate $r$ & $0 \%$ \\
\hline VAT rate & $19 \%$ \\
\hline
\end{tabular}

Calculation of levelized cost $\mathrm{LCoH}$ :

Where:

$$
L C o H=\frac{I_{0}-S_{0}+\sum_{t=1}^{T} \frac{C_{t}(1-T R)-D E P_{t} \cdot T R}{(1+r)^{t}}-\frac{R V}{(1+r)^{T}}}{\sum_{t=1}^{T} \frac{E_{t}}{(1+r)^{t}}}
$$

$\mathrm{LCoH}$ : levelized cost of heat in $€ / \mathrm{kWh}$

$I_{0}$ : initial investment in $€$

$\mathrm{S}_{0}$ : subsidies and incentives in $€$

$C_{t}$ : operation and maintenance costs (year $\mathrm{t}$ ) in $€$

$T R$ : corporate tax rate in $\%$
$D E P_{t}$ : asset depreciation (year $\mathrm{t}$ ) in $€$

$R V$ : residual value in $€$

$E_{t}$ : saved final energy (year $\mathrm{t}$ ) in $\mathrm{kWh}$

$r$ : discount rate in \%

$T$ : period of analysis in years

\section{Annex: Comparison to figures published in Solar Heat Worldwide}

To compare the above presented $\mathrm{LCoHs}$ based on the saved final energy with the $\mathrm{LCoH}_{\text {sHww }}$ presented in Solar Heat World Wide based on the collector yield the following table is presented

\begin{tabular}{|l|l|}
\hline Collector yield (year t) $\mathrm{E}_{\mathrm{t}}$ & $4541 \mathrm{kWh}$ \\
\hline LCoH $_{\text {sHww }}$ solar part without VAT & $\mathbf{0 . 1 2 4} € / \mathrm{kWh}$ \\
\hline
\end{tabular}

\section{References}

[1] EN 12977-2 (2012): “Thermal solar systems and components - Custom built systems - Part 2: Test methods for solar water heaters and combisystems".

[2] COMMISSION DELEGATED REGULATION (EU) No 812/2013, ANNEX VII.

[3] Hafner, B. (2016): “E-Mail“. Dated 13.06.2016.

[4] Check24 (2016): “Würzburg reference costs”. URL: www.check24.com (accessed in Sept. 2016).

[5] Mean values of evaluated invoices, supplied by Bafa.

[6] Louvet, Y., Fischer, S. et. al. (2017): "IEA SHC Task 54 Info Sheet A1: Guideline for levelized cost of heat (LCOH) calculations for solar thermal applications". URL: http://task54.iea-shc.org/. 
[7] Louvet, Y., Fischer, S. et.al. (2017): “Entwicklung einer Richtlinie für die Wirtschaftlichkeitsberechnung solarthermischer Anlagen: die LCOH Methode". Symposium Thermische Solarenergie, Bad Staffelstein.

\footnotetext{
${ }^{1}$ To avoid confusion with the results of other works ([1], [8], [9]) also using the notion of LCoH for solar thermal systems, new acronyms were introduced in this Info Sheet. As previous studies have considered different assumptions for the definition of the terms of the LCOH equation, it does not make sense to compare the values they obtained with the $\mathrm{LCoHs}, \mathrm{LCoHc}$ and $\mathrm{LCoHo}$ values defined here. A detailed explanation of the differences between the approaches chosen in the framework of IEA-SHC Task 54 and in the Solar Heat Worldwide report [9] can be found in Info Sheet A13 [10].
} 